CAHIERS DE

NARRATOLOGIE

\section{Cahiers de Narratologie}

Analyse et théorie narratives

$38 \mid 2020$

Lusor in Fabula. Jeu vidéo et nouvelles frontières du récit

\title{
Rebel without a choice
}

Portal/Portal 2, ou comment raconter l'insubordination dans un jeu à structure linéaire contraignante

\section{Mélanie Bost-Fievet}

\section{OpenEdition}

\section{Journals}

Édition électronique

URL : http://journals.openedition.org/narratologie/11571

DOI : $10.4000 /$ narratologie. 11571

ISSN : 1765-307X

Éditeur

LIRCES

Référence électronique

Mélanie Bost-Fievet, « Rebel without a choice », Cahiers de Narratologie [En ligne], 38 | 2020, mis en

ligne le 11 janvier 2021, consulté le 29 janvier 2021. URL : http://journals.openedition.org/narratologie/ 11571 ; DOI : https://doi.org/10.4000/narratologie.11571

Ce document a été généré automatiquement le 29 janvier 2021.

Article L.111-1 du Code de la propriété intellectuelle. 


\title{
Rebel without a choice
}

\author{
Portal/Portal 2, ou comment raconter l'insubordination dans un jeu à \\ structure linéaire contraignante
}

Mélanie Bost-Fievet

1 Portal $^{1}$ et Portal $2^{2}$ sont des jeux d'énigme en vue à la première personne dans lesquels le joueur incarne un personnage mutique, que le jeu ne nomme jamais directement et que l'on n'aperçoit que furtivement: Chell. Pas d'armes, pas de barre de vie, le joueur peut uniquement ramasser des objets, sauter, et surtout, ouvrir des portails à l'aide d'un générateur à portails (en anglais, portal gun : le design et le nom de l'objet, de même que le gameplay pour viser et « tirer » Marc Marti2020-10-31T12:01:00MM(souris sur PC, commandes manette sur PS3 et Xbox 360), évoquent bien l'arme de tir familière de tous les joueurs de FPS). Un portail bleu, un portail orange, et l'on passe instantanément de l'un à l'autre afin de se déplacer dans toutes les dimensions de l'environnement, d'y réussir les « tests » proposés, salle après salle.

Dans le premier opus, Chell se réveille dans une cellule de relaxation hermétiquement close, de laquelle elle sort lorsqu'un portail s'ouvre dans l'un des murs. Elle progresse alors à travers une série de salles de tests, résolvant des énigmes de plus en plus difficiles pour atteindre la sortie, à l'aide du portal gun, de cubes pouvant servir de poids ou de marches, et des lois de la cinétique. À mesure qu'elle avance, des dangers apparaissent dans les salles: sol couvert d'un liquide toxique, tourelles de défense prêtes à mitrailler tout intrus, boules d'énergie qui pulvérisent tout ce qu'elles touchent. Il n'y a personne dans le «Centre d'enrichissement Aperture », hormis la voix de l'IA qui encourage Chell, l'avertit des dangers qui l'attendent ou la félicite de ses succès. Les messages de l'IA, dans les premières phases du jeu, paraissent préenregistrés, mais petit à petit, ils se font plus personnalisés, jusqu'à constituer une véritable interaction en réponse aux actions spécifiques de Chell. Le jeu bascule lorsque cette dernière triomphe du dernier test: loin de la récompense promise, une plateforme la conduit droit vers un brasier... Une nouvelle phase de jeu s'ouvre alors, dans laquelle Chell navigue dans les coulisses décaties du Centre, cherchant à s'évader à l'aide du portal gun, avant d'affronter finalement l'IA meurtrière, GLaDOS ${ }^{3}$, qui avait voulu se débarrasser d'elle. Sa victoire contre le «boss » est de courte durée : Chell se 
retrouve bien à l'extérieur du Centre, mais sonnée par l'explosion, et il ne se passe que quelques secondes avant que des robots ne viennent la tirer à nouveau dans les profondeurs d'Aperture, tandis que se déclenche un générique où la voix de GLaDOS, narquoise, lui chantonne qu'elle, elle est toujours vivante ( (Still alive $\left.{ }^{4} »\right)$.

Le second opus commence une éternité plus tard. Chell a été placée en biostase pendant un temps indéterminé, et le Centre Aperture a commencé à tomber en ruine. C'est un nouveau robot, Wheatley, qui réveille Chell, révélant à l'occasion qu'elle est probablement la seule survivante parmi les milliers de patients en biostase. Le Centre est sur le point d'exploser, et Wheatley, guère compétent, s'efforce d'aider Chell à se mettre en sécurité. Ce faisant, ils réveillent involontairement GLaDOS, bien vivante et verte de rage contre Chell ; l'IA sépare les deux personnages et recommence aussitôt à créer des salles de test pour torturer sa cobaye préférée... Wheatley parvient cependant à rejoindre Chell et à priver GLaDOS de tout moyen de nuire, en neutralisant les tourelles et la neurotoxine qu'elle utilisait dans le premier épisode. Il convainc ensuite Chell de le mettre aux commandes à la place de GLaDOS, dont il transfère l'IA dans une pile-pomme de terre. Toutefois, ce pouvoir tout neuf monte à la tête de Wheatley, qui se transforme instantanément en grand méchant mégalomane. Ainsi s'ouvre la seconde phase de jeu, avec un changement d'antagoniste: d'abord dans les ruines du centre Aperture, où Chell s'efforce d'évoluer en découvrant de nouveaux tests basés sur des gels (un accélérateur, un gel rebondissant, et une substance lui permettant de créer des surfaces supports de portails), puis dans les salles de test que Wheatley - sans grand talent ni intelligence - lui bricole. Chell progresse ainsi, avec l'aide d'une GLaDOSpatate bien déterminée à se venger du robot imbécile qui l'a évincée. Elle parvient enfin à affronter cette deuxième IA meurtrière dans une boss battle évocatrice de la première et qui en a retenu les leçons. GLaDOS réintègre enfin sa structure... puis décide de se débarrasser de celle qui lui a rendu la vie si compliquée, parce que la tuer, vraiment, c'est trop d'effort. Chell est éjectée du centre Aperture, se retrouve dans un champ de maïs au milieu de nulle part, près d'une bicoque perdue dont la porte se claque dans son dos : c'est la fin.

4 Portal et Portal 2 sont des jeux à solution unique : pas d'itinéraire alternatif possible l'exploration de salles cachées ne sert qu'à fournir des easter eggs, elle ne change rien au déroulement -, pas même de dialogue, bref, pas de choix. La bonne solution, c'est toujours la seule solution possible, que le joueur se trouve dans les phases de «tests » ou d'évasion. Et pourtant, GLaDOS rappelle sans cesse à Chell qu'elle est une rebelle, obstinée, destructrice, qui brise tout (et surtout son cœur). Tout le jeu réaffirme sans cesse au joueur qu'il est différent, qu'il s'échappe, qu'il s'émancipe, alors qu'il ne lui en offre aucune possibilité réelle. Qu'est-ce qui permet, alors, au joueur de jouer les rebelles? Comment soutenir une narration apparemment contradictoire avec les mécanismes du jeu? Deux stratégies, principalement: un monde qui fait un usage délicieux des paradoxes et dissonances, et une narration qui interroge avec intelligence le rapport au jeu et à la récompense.

\section{Know your paradoxes!}

Dès le commencement, l'esthétique des deux opus repose sur une tension constante entre un humour volontiers fantasque et une noirceur merveilleusement cruelle. Deux musiques se superposent au début du premier Portal: un thème extra-diégétique 
menaçant et sourd, et des radios intra-diégétiques qui jouent une mélodie d'ascenseur aussi entraînante qu'irritante ${ }^{5}$. Deux ambiances visuelles, ensuite, dans les décors : d'un côté, des salles de test impeccables, d'une blancheur clinique, de l'autre des coulisses manifestement abandonnées, en désordre, meubles renversés, caméras dont plus personne ne regarde les images, écrans d'ordinateurs affichant des messages d'erreur, ainsi que des salles cachées recouvertes des graffitis inquiétants d'un personnage inconnu, visiblement dérangé6. Idem pour les accessoires du jeu, visibles et horschamp : leur désignation rappelle les circonvolutions bureaucratiques les plus tordues, systématiquement précédées de la marque "Aperture science» (Aperture science weighted storage cube, Aperture science self-esteem fund for girls...), jusqu'à des sommets d'absurdité atteints avec un objet appelé par GLaDOS «Aperture science we dont know what it is ». Cette anaphore comique peut fonctionner comme un clin d'œil à l'humour cartoon et à la marque "Acme » accolée à tous les accessoires improbables qui permettent au Coyote de dresser ses stratagèmes - après tout, la situation d'un joueur débutant n'est pas très loin de celle d'un Coyote : Die and retry! Enfin, chaque salle est accompagnée de messages audio de l'IA, qui marient la plus absconse rhétorique bureaucratico-scientifique, affirmant avec insistance le souci de sécurité et de sérieux du Centre, et, derrière le jargon et les euphémismes lénifiants, la révélation quasi-systématique d'une menace de mort. Les sujets des salles de tests sont ainsi poliment avertis que les effets secondaires peuvent comporter des «permanent disabilities, such as vaporization », et qu'un échec les exposerait à «an unsatisfactory mark on your record, followed by death ».

6 Ces contrastes dans l'univers sonore et visuel n'ont pas seulement pour fonction d'élaborer une atmosphère d'humour noir - même si cet humour noir est, à n'en pas douter, un des éléments qui fait le charme et le succès du jeu. Très tôt dans le jeu, les messages entendus mettent en œuvre une dissonance entre consigne explicite et consigne réelle, et alertent le joueur : le jeu leur ment, ou plus exactement, « améliore la réalité » (enhances reality) dans le cadre du " protocole ». En arrivant dans l'une des premières salles du jeu, la voix nous avertit : le prochain test est cassé ; il est impossible à résoudre ; abandonnez tout de suite et vous aurez du gâteau, répète la voix - sauf que... non, continue le message une fois que le joueur a tout de même résolu le test, il s'agissait seulement de tester sa réaction « in an atmosphere of extreme pessimism ». La salle en question offre une énigme aisée, et le level design fait que le joueur n'hésite guère quant à sa capacité à la résoudre, si bien qu'immédiatement il cesse de croire à ce que dit la voix, qui semble alors réduite à un pur ornement comique. Toujours est-il que l'usage du mensonge est établi très tôt comme une règle du jeu. Le statut même de ces messages, d'ailleurs, est trompeur. Ils ont toute l'apparence de messages préenregistrés, parfaitement impersonnels (s'adressant au joueur comme "you, [insert subject's name here]»), grevés de dysfonctionnements sonores - le son déraille, part dans les aigus ou les graves. Et puis leur contenu parait absurde, relevant du pur comique : ça n'a aucun sens, «You will be baked, and then there will be cake »! À moins que ce ne soit exactement ce qui va se passer : la récompense qui attend Chell est bien un brasier... Car en dépit de leurs apparences trompeuses, ces messages ne sont pas pré-enregistrés, l'IA s'adresse bien à Chell (ou à nous), et tout le reste est mise en scène, manipulation. Mensonge ludique, mensonge métaludique: le joueur nage en pleine dissonance. 
7 L'un des meilleurs exemples de dissonance ludonarrative intervient assez tôt dans le premier épisode, avec le «Cube de voyage» (weighted companion cube), un cube lesté orné d'un joli petit cœur rose sur chacune de ses faces, et que le joueur doit conserver pour plusieurs usages dans la salle qui suit, alors qu'auparavant les cubes avaient un usage ponctuel. Des messages de l'IA sont martelés pour le dissuader de s'y attacher excessivement, lui rappeler qu'il s'agit d'un objet inanimé - et, paradoxalement, donc, pour suggérer au joueur que s'y attacher est une possibilité plausible, une idée renforcée par la découverte d'une salle secrète ornée de poèmes d'amour à sa gloire, laissés là par le mystérieux auteur des graffitis cachés un peu partout dans le jeu ${ }^{7}$. À la fin de la salle, quand vient le moment d'incinérer le cube, le joueur peut hésiter : faut-il maintenant défier la consigne? Il faut bien s'y résoudre, sans quoi la progression est impossible; de toute façon qu'il hésite ou pas, le message sera le même, annonçant que le joueur a battu un record en l'incinérant (la voix dit « euthanasiant ») à toute vitesse. C'est le métadiscours qui crée à lui seul le dilemme moral, qui l'invente.

Comme le joueur comprend vite que le jeu le manipule, la désobéissance devient vite une seconde nature - et le jeu s'en sert. Dans le second épisode, GLaDOS dissuade Chell d'emporter avec elle un cube ordinaire, et l'unique motivation du joueur à faire le contraire est la défense qui lui est formulée : il n'a jamais auparavant été nécessaire de transporter du matériel au-delà des sas qui séparent les salles, c'est une règle tacite du jeu. Sauf que le cube se fait bien désintégrer, et GLaDOS, impitoyable, nous culpabilise en expliquant que ces cubes sont doués de sensibilité, «of course ». GLaDOS est, de toute évidence, une experte de la psychologie inversée et de la manipulation, et de nombreux moments peuvent se lire comme des exemples de game theory perverse, notamment le «boss" final du premier épisode: GLaDOS répète avec une telle insistance à Chell de ne pas toucher au module qui est tombé qu'il est immédiatement évident que c'est ce qu'il faut faire pour progresser. Or la destruction de ce module élimine les barrières morales de GLaDOS, qui inonde aussitôt la pièce de neurotoxine, donc peut-être que c'était bien de la psychologie inversée, un plan machiavélique pour avoir enfin les mains libres, en comptant sur le réflexe de désobéissance acquis, à ce stade, par le joueur...

9 Toute consigne donnée est ainsi reçue comme une invitation à la transgression. D'ailleurs, ne pas appliquer les consignes est souvent la solution manifeste: c'est évidemment le cas lorsque Chell est invitée, à la fin du premier épisode, à s'allonger sur le sol, les bras le long du corps, dans une position de "party escort submission». Et dans Portal 2, rien de tel pour flécher la bonne direction que de surcharger l'écran de panneaux « Keep out/Do not enter ». Mais le jeu est parfois plus subtil et s'amuse avec les limites de ses propres mécaniques. Au début du second épisode, pour tester les fonctions vitales de Chell, Wheatley lui demande de parler, tandis que le message écrit de tutoriel à l'écran indique de sauter. Comme de toute façon parler n'est jamais une option, le joueur exécute la commande extra-diégétique, et Wheatley émet l'hypothèse d'une atteinte cérébrale. C'est à la fois la «bonne » et la "mauvaise » réponse, et le jeu fonde une grande partie de sa dynamique et de son humour sur cette tension.

Car Portal et Portal 2 adorent les paradoxes, au point d'en faire un élément de gameplay et de narration, et même une arme. Dans le second opus, GLaDOS envisage de vaincre Wheatley en lui proposant un paradoxe à méditer, après avoir vu un poster dans l'une des salles donnant des consignes pour résister à une IA rebelle: lui soumettre un paradoxe, par exemple affirmer « Cette phrase est fausse », car aucune IA 
ne peut résister à un paradoxe, c'est bien connu ! Malheureusement, Wheatley est trop bête pour que le plan fonctionne (GLaDOS, elle, manque d'y rester). Et le comic Lab Rat accumule les allusions au chat de Schrödinger : l'état de stase où Chell est laissée, seule et sans personne pour veiller sur elle, est incertain, si bien qu'elle est peut-être morte, peut-être pas. Know your paradoxes! Face à des IA devenues rogues, ils sont votre meilleure chance - à moins que ce ne soit l'IA, évidemment, qui nous promène depuis le début de paradoxe en paradoxe...

11 Car le plus grand des paradoxes, bien sûr, c'est l'IA elle-même, qui tient à la fois le rôle d'adjuvant et d'opposant. GLaDOS le rappelle elle-même lors du premier combat final : «Killing you and giving you good advice aren't mutually exclusive» - elle semble parfaitement sincère en l'affirmant, et dans une certaine mesure, c'est l'exacte vérité. Le jeu, qu'elle incarne, a deux dynamiques : tuer et guider. Or c'est GLaDOS qui crée chacune des salles de tests, ainsi que leur succession: son action est rendue évidente dans le second opus, où l'on assiste en direct à leur création puisque le Centre a été en partie détruit et qu'elle doit tout réparer. En d'autres termes, elle assure le level design de chaque salle de test; elle est bien ce qui rend le jeu possible (du point de vue du joueur), mais aussi ce qui l'entrave. De surcroît, tout son personnage est écrit avec un talent indéniable pour le chantage affectif, alternant entre la lamentation culpabilisante alors qu'elle a essayé plusieurs fois de tuer Chell (« What have I ever done to you ?", "The only thing you managed to break is my heart ", " Have I lied to you? I mean, in this room?", "Who murders people who are only trying to help them ? Nobody is that cruel ») et la vacherie passive-agressive (elle félicite Chell d'être le seul sujet qui ait réussi à prendre du poids dans la chambre de stase, fait régulièrement référence au fait que ses parents l'ont abandonnée, qu'elle a été adoptée et qu'elle n'a pas d'amis, et de la façon la plus glaciale et protocolaire qui soit : « Shall not be mourned. It says so in your file. Very formal, very official. »)

Mais cela va plus loin. GLaDOS fait exister le jeu, ses embûches, mais aussi les conditions de la rébellion contre lesdites embûches et contre le jeu. Comme Chell ne peut pas parler, et que le joueur n'a pas d'autre choix que d'évoluer dans le jeu, que ce soit dans les salles de test (le jeu construit par GLaDOS puis par Wheatley) ou dans les coulisses du Centre (hors du parcours prévu et construit par les deux IA), ce qui nous assure qu'elle se rebelle, ce sont les lignes de dialogue de GLaDOS. C'est elle qui accuse Chell, à la fin du premier opus et dans la première moitié du second, d'avoir tout gâché, d'être une fauteuse de troubles ; c'est elle encore qui, lorsqu'elle devient son alliée à la moitié de Portal 2, exprime à grand bruit sa décision à elle de se rebeller à son tour. Sans son discours, le joueur ne fait que suivre le seul chemin possible; il n'intériorise l'idée du choix et de la révolte que parce que l'alternative, sa soumission, est mise en scène de l'extérieur comme un choix - parce que le jeu lui affirme après coup qu'il n'était pas obligé de choisir ce qu'il a choisi, même après l'avoir contraint. Mais on n'est plus à une manipulation, ni à un paradoxe près...

\section{And then there will be cake}

Donc, Portal est un jeu à progression contrainte, déguisé en jeu à choix. Mais est-ce qu'il est possible de gagner à Portal ? Est-ce qu'il est possible de ressentir le frisson de la récompense? 
qui est devenue probablement la plus connue des références geek au jeu : CAKE. «Cake will be served ", promet presque tout de suite la voix de GLaDOS, relayée ensuite par des pictogrammes représentant une savoureuse part de gâteau (un message précise " cake and grief counseling will be available at the end of the test ", des fois que l'on serait trop optimiste). Le gâteau-récompense est une véritable obsession dans tout le jeu, revenant dans la chanson de générique (« you just keep on trying til you run out of cake ») puis dans le post-générique qui montre enfin le gâteau promis, avec un comique de répétition d'un absurde aussi savoureux que cette inaccessible pâtisserie. Sauf que, comme tout amateur de memes vidéoludiques le sait - et si on l'ignore encore, les graffitis effrayants des salles cachées se feront une joie de le lui apprendre : The cake is a lie9. Pourtant, le rapport du jeu à la mécanique de récompense est plus compliqué que cela.

D'abord, le jeu surjoue comiquement le renforcement positif, inondant de compliments le joueur cobaye - que ce soit GLaDOS dans le premier opus, ou, dans une phase du second, Cave Johnson, le grand fondateur d'Aperture Science, dont les messages préenregistrés félicitent chaudement les volontaires dévoués pour la science, avec l'enthousiasme et la fermeté d'un adjudant face à ses pauvres recrues. Ladite "science» est constamment invoquée comme justification de tout le protocole expérimental, au point que le terme se retrouve totalement désémantisé. C'est le cas chez tous les locuteurs d'Aperture, que ce soit GLaDOS («there's science to do », " the science gets done »), les messages impersonnels du début de Portal 2, et surtout ceux de Cave Johnson, qui en sature son discours avec une totale décontraction («we're just throwing science at the wall to see what sticks ", " why don't you marry safe science if you love it so much »). L'absurdité monte d'un cran au début de Portal 2, puisque les messages pré-enregistrés prennent acte d'une situation apocalyptique mais se réjouissent que, malgré tout, les tests puissent se poursuivre (avec quelques précautions d'usage : «if the laws of physics no longer apply in the future... God help you »). Au final, hormis cette " science » à la fois sacralisée et vidée de tout contenu, les tests n'ont jamais d'autre but qu'eux-mêmes : faire subir des tests, encore des tests, à la seule fin de tester.

Ainsi, chacune des «récompenses" verbales est en réalité l'occasion de ramener le personnage à son statut déshumanisé de cobaye. Son nom n'est pas donné puisqu'il s'agit de messages-types, dont l'impersonnalité est même exhibée par le maintien de mentions vouées à être remplacées («you, [insert subject name here], must be the pride of [insert subject's hometown here] ») ou par des mises en garde rappelant que les messages ne font que spéculer sur les performances et la réussite du joueur (" please disregard any undeserved compliments»). Ce statut de cobaye anonyme et déshumanisé recouvre celui du joueur : n'être là que pour résoudre des énigmes, sans qu'un autre but ne soit véritablement apparent. La rébellion du joueur, finalement, comme celle de Chell, ce sera d'exiger un but, qui ne soit pas uniquement de passer au niveau suivant, ou d'échapper à la mort. Cette déshumanisation est renforcée par le brouillage des frontières entre les différentes machines ou IA et les humains. Les prothèses que porte Chell aux jambes, sa biostase prolongée, lui confèrent des aspects transhumains; de leur côté, les robots, même les plus basiques, sont dotés d'une affectivité, puisque les tourelles de défense murmurent un triste I don't blame you lorsqu'elles sont détruites, communiquent musicalement, comme le révèle le post-

Cahiers de Narratologie, 38 | 2020 
générique de Portal 2, et ont peut-être même une âme immortelle (« Android hell is a real place", nous rappelle GLaDOS). Le mode multiplayer de Portal 2 ne fait que confirmer ce que GLaDOS suggère dans le jeu, c'est-à-dire que Chell peut parfaitement être remplacée par des androïdes, qui pourraient satisfaire la pulsion de test de l'IA sans l'encombrer des velléités de révolte humaines.

On apprend donc très vite à ne pas se fier au renforcement positif des messages préenregistrés de félicitations impersonnelles, et à en rechercher un autre, bien familier des joueurs : le simple déclenchement d'un dialogue quel qu'il soit, qui confirme que la voie empruntée est la bonne. Portal joue abondamment avec ce code, par une utilisation ludique de l'antiphrase: dans la phase d'évasion, on s'habitue à entendre comme autant de félicitations toutes les assertions de GLaDOS assénant qu'on ne va pas dans la bonne direction, répétant, à chaque réussite, "You haven't escaped, you know ", ou encore "You're not even going the right way ». Mais si le stratagème est efficace, il constitue aussi, sournoisement, une première manière de voler un peu au joueur la sensation de la récompense.

Dans les salles de test, ladite récompense devrait être, tout simplement, d'avoir trouvé la solution et atteint le sas qui doit conduire à la prochaine salle. Ah, la satisfaction d'avoir enfin résolu une énigme difficile... Mais satisfaction pour qui, au juste? Pas pour Chell, apparemment, et peut-être même pas pour le joueur. C'est dans Portal 2 que cet enjeu devient évident: lorsque Wheatley passe aux commandes, il exprime bruyamment l'extrême plaisir qu'il tire de chaque réussite à un test, avec des râles de plaisir (tout joueur confirmé sait bien que notre cerveau adore que l'on chatouille son circuit de la récompense). Sensation fugitive, hélas, puisque bien vite Wheatley y développe une accoutumance telle qu'il ne parvient plus à l'éprouver à nouveau, et c'est alors qu'il faut recourir aux grands moyens : insultes d'abord, menaces de mort pas très longtemps après, et exécution, bien sûr.... Non seulement les cobayes ne reçoivent jamais la satisfaction que leur dévouement ait du sens - tout étant fait au nom de la "science » comiquement déifiée - mais même le plaisir de la réussite est détourné par les IA qui se l'approprient. Pour retrouver une forme d'agentivité, le joueur doit reconquérir le plaisir de la récompense, un des éléments les plus évidents, mais les moins souvent mis en scène dans les jeux.

Retour à la question, donc : peut-on gagner à Portal ? À la fin du premier épisode, Chell passe peut-être cinq secondes dehors, à peine consciente, avant d'être à nouveau traînée dans les profondeurs du Centre où elle restera pendant une éternité, condamnée à un avenir post-apocalyptique; le boss, GLaDOS, n'est pas morte mais, comme elle le susurre, "still alive ", et apparemment immortelle ${ }^{10}$. Drôle de victoire... Quant au second opus, Chell le termine libre et dehors, c'est vrai... mais après que GLaDOS l'a éjectée, et non de sa propre volonté. Si GLaDOS lui annonce "You win ", c'est plutôt avec le ton d'un parent excédé mais persuadé que l'enfant rebelle souffrira les conséquences de son obstination. Elle renonce à tuer Chell uniquement parce qu'elle veut s'en débarrasser, et que l'éjecter est définitivement plus facile que de la tuer: "Want you gone", nous chantonne cette fois le générique. Les paroles dépeignent la condition humaine et la liberté comme bien peu désirables et assez minables ${ }^{11}$. Drôle de victoire encore, qui n'est l'objet que de moqueries, et qui ne fait que réaffirmer l'indéboulonnable toute-puissance de l'IA rancunière à laquelle on n'échappe que parce qu'elle préfère se débarrasser d'une nuisance... À moins que l'on ne considère que dans 
un jeu qui brille par ses paradoxes, son ironie et ses références métaludiques, les génériques en question ne constituent la récompense absolue.

Le bon chemin, dans Portal, est systématiquement le seul chemin possible. Pourtant, le suivre est mis en scène comme un choix : " you chose this path ", affirme GLaDOS. Dans Portal 2, Chell est forcée de commettre l'erreur qui va mettre Wheatley aux commandes, puis de la réparer : il n'y a pas d'alternative - un précepte qui convient fort bien à un univers de firme toute-puissante, déshumanisante et qui joue à Dieu... Pour que Chell soit spéciale, il faut faire exister virtuellement d'autres humains : les malheureux cobayes évoqués par les messages pré-enregistrés, l'auteur des graffitis psychotiques qui guident Chell vers la sortie, promesse d'une altérité humaine dont l'humanité rejaillit, un tant soit peu, sur Chell ${ }^{12}$. Ces signaux auditifs et visuels racontent l'existence de trajectoires qui ne sont pas, ou pas tout à fait, la sienne et celle du joueur : dès lors, il peut $\mathrm{y}$ avoir un choix et une victoire, au moins par rapport à ces voies alternatives dont on devine l'échec à l'absence de ceux qui les ont frayées.

Quant à GLaDOS, ouvrière du jeu-test, garante de la progression du joueur à mesure même qu'elle l'entrave, humaine devenue IA, elle fonctionne certainement comme une métaphore du game designer. Dans "Still alive ", ne chante-t-elle pas "We're out of beta, we're releasing on time » - une phrase qui n'a aucun sens dans le contexte de la diégèse ? The Stanley Parable ${ }^{13}$ est de ce point de vue un héritier naturel de Portal et Portal 2, jouant sur la tension entre la liberté du joueur et les consignes du " narrateur ", autre avatar du game designer, qui, en lui disant où aller, l'invite en fait à désobéir et à explorer d'autres trajectoires et d'autres fins, prolongeant la réflexion sur le libre-arbitre et la relation du joueur à son jeu. Toutefois, The Stanley Parable est un jeu à choix, où un éventail de fins est proposé selon les différentes formes de rébellion essayées par le joueur, là où Portal et Portal 2 n'offrent qu'une seule fin possible, bien moins métaphysique ou délirante que les plus baroques explorées par Stanley. Mais face à la mode des arborescences de choix vertigineuses dont certaines se révèlent bien superficielles, le ton délicieusement sarcastique avec lequel Portal, d'un même mouvement, nous fait miroiter la petitesse de nos victoires vidéoludiques et s'évertue à nous construire un personnage de rebelle au cœur même de l'absence d'alternative, sonne comme l'impossible réponse aux paradoxes qui torturent les IA.

\section{NOTES}

1. Portal, Valve, 2007, conçu par Erik Wolpaw, Chet Faliszek et Kim Swift.

2. Portal 2, Valve, 2011, réalisé par Joshua Weier, scénarisé par Erik Wolpaw, Jay Pinkerton et Chet Faliszek, produit par Gabe Newell.

3. GLaDOS est l'acronyme de Genetic Lifeform and Disk Operating System.

4. "Still alive", écrite par Jonathan Coulton et chantée par Ellen McLain, la comédienne qui assure le doublage de GLaDOS.

5. Mélodie qui sera d'ailleurs reprise dans le générique de fin, « Still Alive ». 
6. L'identité de ce personnage demeure mystérieuse dans les jeux, mais elle est révélée dans le comic Lab Rat (illustré et scénarisé par Michael Avon Oeming and Jay Pinkerton, publié par Valve en 2011, disponible en ligne). Le scientifique Doug Rattmann est l'unique survivant du massacre opéré par GLaDOS, et il est révélé qu'il veille sur Chell à mesure qu'elle progresse dans ce qui constitue la trame du premier jeu, avant de venir à son secours lorsqu'elle est récupérée par les robots et de la mettre en état de biostase. Rattmann est manifestement schizophrène et tourmenté par des hallucinations.

7. Et même des photos de pin-up dans lesquelles le visage a été remplacé par un collage du cube.

8. En réaction enthousiaste à l'accès de fureur de Cave Johnson, à qui on a demandé de ne plus enregistrer de messages, et ne l'entend pas de cette oreille: "When life gives you lemons? Don't make lemonade. Make life takes these lemons back! Get mad! I don't want your damn lemons! (...) Demand to see life's manager!»

9. La phrase "The cake is a lie » a été détournée dans de nombreux memes, d'abord comme un clin d'œil dans la communauté des joueurs, puis avec un sens allégorique dépassant Portal - à la grande exaspération de quelques "vrais gamers " autoproclamés qui se sentaient dépossédés de leur référence, et même du créateur du jeu, Erik Wolpaw, qui décida de supprimer toute référence dans le second opus (voir par exemple les pages https://www.dictionary.com/e/ memes/the-cake-is-a-lie/ et https://knowyourmeme.com/memes/the-cake-is-a-lie, consultées le 26.06.20).

10. «When you're dying I will be still alive/ and when you're dead I will be still alive. »

11. "You want your freedom? Take it ", "You've got your short sad life left (...) I'll let you right back to it. "

12. On peut, à cette occasion, mentionner les théories de fans sur les origines de Chell, appuyées sur des indices disséminés par le jeu, comme les mentions d'un «Bring your daughter to work day " et la présence de son prénom à côté d'une expérience de pile-pomme de terre, ainsi que par Lab Rat et par le site www.aperturescience.com. Chell est parfois donnée comme la fille de Cave Johnson et de sa secrétaire, Caroline, première cobaye dont l'esprit est transféré dans une IA et qui devient nulle autre que... GLaDos.

13. The Stanley Parable (D. Wreden et W. Pugh, Galactic Cafe, 2011) a initialement été produit comme un mod de Half-Life 2, jeu également produit par Valve en 2004, et dont l'univers recoupe celui des deux Portal - Black Mesa, la firme centrale dans la licence Half-Life, est la grande rivale d'Aperture, mentionnée comme un easter egg à plusieurs reprises dans Portal. Dans le walking simulator The Stanley Parable, le joueur incarne un médiocre employé, Stanley, dont un narrateur raconte la journée. Libre à lui, alors, d'agir en reproduisant les actions décrites par le narrateur (prendre la porte de droite, monter l'escalier, etc) ou de désobéir, déclenchant des réactions de la part du narrateur et des développements inattendus de l'histoire.

\section{RÉSUMÉS}

Portal et Portal 2 sont des jeux d'énigme n'offrant qu'un seul cheminement possible, alors même que, par la voix de l'IA antagoniste de l'héroïne, ils renvoient du joueur l'image d'un rebelle incontrôlable. Les jeux exhibent un usage assumé des paradoxes, plein d'humour noir dans la construction de la diégèse et des dialogues, qui font écho à une dissonance ludonarrative permanente. Ils amènent à remettre en question le statut même de la récompense et de la 
victoire, mais aussi du choix et du libre-arbitre, face à une instance à la fois opposante et adjuvante, double sarcastique et impitoyable du game designer.

Portal and Portal 2 are puzzle games with only one possible path and outcome, even though the heroin's AI antagonist relentlessly calls her - and the player - an unstoppable rebel. Both games display an unapologetic use of paradoxes, through the world-building and dialogues, brimming with dark humor and echoing the constant ludonarrative dissonance. They bring into question the very nature of reward and victory, as well as of choice and free-will, against an authority that's both opponent and adjuvant, a sarcastic, merciless proxy for the game designer.

INDEX

Keywords : Portal, videogame, puzzle games, game design, videogame narration

Mots-clés : Portal, jeu vidéo, jeu d'énigme, game design, narration vidéoludique

\section{AUTEUR}

\section{MÉLANIE BOST-FIEVET}

Mélanie Bost-Fievet, après une thèse en poétique néo-latine, se consacre à la réception de la culture classique (de l'Antiquité à la Renaissance) dans les œuvres littéraires et ludiques de fantasy et de science-fiction. Enseignante en lycée, elle pratique également la vulgarisation et la traduction, et a publié plusieurs nouvelles de SF. 